Rev. Mat. Iberoamericana 19 (2003), 143-160

\title{
On coincidence of $p$-module of a family of curves and $p$-capacity on the Carnot group
}

Irina Markina

\begin{abstract}
The notion of the extremal length and the module of families of curves has been studied extensively and has given rise to a lot of applications to complex analysis and the potential theory. In particular, the coincidence of the $p$-module and the $p$-capacity plays an important role. We consider this problem on the Carnot group. The Carnot group $\mathbb{G}$ is a simply connected nilpotent Lie group equipped with an appropriate family of dilations. Let $\Omega$ be a bounded domain on $\mathbb{G}$ and $K_{0}, K_{1}$ be disjoint non-empty compact sets in the closure of $\Omega$. We consider two quantities, associated with this geometrical structure $\left(K_{0}, K_{1} ; \Omega\right)$. Let $M_{p}\left(\Gamma\left(K_{0}, K_{1} ; \Omega\right)\right)$ stand for the $p$-module of a family of curves which connect $K_{0}$ and $K_{1}$ in $\Omega$. Denoting by $\operatorname{cap}_{p}\left(K_{0}, K_{1} ; \Omega\right)$ the $p$-capacity of $K_{0}$ and $K_{1}$ relatively to $\Omega$, we show that

$$
M_{p}\left(\Gamma\left(K_{0}, K_{1} ; \Omega\right)\right)=\operatorname{cap}_{p}\left(K_{0}, K_{1} ; \Omega\right)
$$
\end{abstract}

\section{Introduction}

Let $D$ be a domain (an open, connected set) in $\overline{\mathbb{R}}^{n}=\mathbb{R}^{n} \cup\{\infty\}$, and let $K_{0}$, $K_{1}$ be disjoint non-empty compact sets in the closure of $D$. We denote by $M_{p}\left(\Gamma\left(K_{0}, K_{1} ; D\right)\right)$ the $p$-module of a family of curves which connect $K_{0}$ and $K_{1}$ in $D$. Next we use the notation $\operatorname{cap}_{p}\left(K_{0}, K_{1} ; D\right)$ for the $p$-capacity of the condenser $\left(K_{0}, K_{1} ; D\right)$ relatively to $D$. The question about coincidence of the $p$-module of a family of curves and the $p$-capacity for various geometric configuration has been studied by many authors. For example, in the case

2000 Mathematics Subject Classification: Primary 31B15; Secondary 22E30.

Keywords: p-module of a family of curves, $p$-capacity, Carnot-Carathéodory metrics, nilpotent Lie groups. 
when $K_{0}$ and $K_{1}$ do not intersect the boundary of $D$ and either $K_{0}$ or $K_{1}$ contains the complement to an open $n$-ball the problem has been solved affirmatively by Ziemer in [23]. Hesse in [10] has generalized this result requiring only $\left(K_{0} \cup K_{1}\right) \cap \partial D=\emptyset$. In the series of papers [2, 3, 4] Caraman has been studying the problem under various conditions on the tangency geometry of the sets $K_{0}$ and $K_{1}$ with the boundary of $D, D \in \overline{\mathbb{R}}^{n}$. In 1993 Shlyk [16] proved, that the coincidence of the $p$-module and $p$-capacity is valid for an arbitrary condenser $\left(K_{0}, K_{1} ; D\right), K_{0}, K_{1} \in \bar{D}, D \in \overline{\mathbb{R}}^{n}$, $\left(K_{0} \cup K_{1}\right) \cap \partial D \neq \emptyset$.

A stratified nilpotent group (of which $\mathbb{R}^{n}$ is the simplest example) is a Lie group equipped with an appropriate family of dilations. Thus, this group forms a natural habitat for extensions of many of the objects studied in the Euclidean space. The fundamental role of such groups in analysis was envisaged by Stein $[17,18]$. There has been since a wide development in the analysis of the so-called stratified nilpotent Lie groups, nowadays, also known as Carnot groups. In the present article we are studying the problem of the coincidence between the $p$-module of a family of curves and the $p$-capacity of an arbitrary condenser $\left(K_{0}, K_{1} ; \Omega\right), \Omega$ is a bounded domain on the Carnot group. In [12] the identity $M_{p}\left(\Gamma\left(K_{0}, K_{1} ; \Omega\right)\right)=\operatorname{cap}_{p}\left(K_{0}, K_{1} ; \Omega\right)$ was proved for the condenser $\left(K_{0}, K_{1} ; \Omega\right)$ on the Heisenberg group, which is a two-step Carnot group, requiring that the compacts $K_{0}$ and $K_{1}$ are strictly inside the domain $\Omega$. We would like to mention the result by Heinonen and Koskela [8] which states that on every general metric spaces the $p$-capacity coincides with the $p$-module but in comparison with our paper they used different definitions. The use of this general result [8] for the Carnot groups requires the fact that the smallest very weak upper gradient of a Lipschitz function is given by the horizontal gradient (see for instance [9]). However it is not clear that the result of [8] covers the case when the intersection of the compacts $K_{i}, i=0,1$, with the boundary of $\Omega$ is not empty. Moreover the case when $\Omega$ is not $\varphi$-convex is not obtained from [8].

The author would like to acknowledge Serguei Vodop'yanov for his helpful remarks and observations.

\section{Notation and definitions}

The Carnot group is a connected, simply connected nilpotent Lie group $\mathbb{G}$, whose Lie algebra $\mathcal{G}$ splits into the direct sum of vector spaces $V_{1} \oplus V_{2} \oplus$ $\ldots \oplus V_{m}$ which satisfy the following relations

$$
\begin{aligned}
{\left[V_{1}, V_{k}\right] } & =V_{k+1}, \quad 1 \leq k<m, \\
{\left[V_{1}, V_{m}\right] } & =\{0\}
\end{aligned}
$$


We identify the Lie algebra $\mathcal{G}$ with the space of left-invariant vector fields. Let $X_{11}, \ldots, X_{1 n_{1}}$ be a bases of $V_{1}, n_{1}=\operatorname{dim} V_{1}$, and let $\langle\cdot, \cdot\rangle$ be a left-invariant Riemannian metric on $V_{1}$ such that

$$
\left\langle X_{1 i}, X_{1 j}\right\rangle= \begin{cases}1 & \text { if } i=j, \\ 0 & \text { if } i \neq j .\end{cases}
$$

Then, $V_{1}$ determines a subbundle $H T$ of the tangent bundle $T \mathbb{G}$ with fibers

$$
H T_{x}=\operatorname{span}\left\{X_{11}(x), \ldots, X_{1 n_{1}}(x)\right\}, \quad x \in \mathbb{G} .
$$

We call $H T$ the horizontal tangent bundle of $\mathbb{G}$ with $H T_{x}$ as the horizontal tangent space at $x \in \mathbb{G}$. Respectively, the vector fields $X_{1 j}, j=1, \ldots, n_{1}$, we will call the horizontal vector fields.

Next, we extend $X_{11}, \ldots, X_{1 n_{1}}$ to an orthonormal basis

$$
X_{11}, \ldots, X_{1 n_{1}}, X_{21}, \ldots, X_{2 n_{2}}, \ldots, X_{m 1}, \ldots, X_{m n_{m}}
$$

of $\mathcal{G}$. Here each vector field $X_{i j}, 2 \leq i \leq m, 1 \leq j \leq n_{i}=\operatorname{dim} V_{i}$, is a commutator

$$
X_{i j}=\left[\ldots\left[\left[X_{1 k_{1}}, X_{1 k_{2}}\right] X_{1 k_{3}}\right] \ldots X_{1 k_{i}}\right]
$$

of length $i-1$ generated by the basis vector fields of the space $V_{1}$.

It was proved (see, for instance, [6]) that the exponential map exp:G $\rightarrow \mathbb{G}$ from the Lie algebra $\mathcal{G}$ into the Lie group $\mathbb{G}$ is a global diffeomorphism. We can identify the points $q \in \mathbb{G}$ with the points $x \in \mathbb{R}^{N}, N=\sum_{i=1}^{m} \operatorname{dim} V_{i}$, by the rule $q=\exp \left(\sum_{i, j} x_{i j} X_{i j}\right)$. The collection $\left\{x_{i j}\right\}$ is called the coordinates of $q \in \mathbb{G}$. The number $N=\sum_{i=1}^{m} \operatorname{dim} V_{i}$ is the topological dimension of the Carnot group. The biinvariant Haar measure on $\mathbb{G}$ is denoted by $d x$; this is the push-forward of the Lebesgue measure in $\mathbb{R}^{N}$ under the exponential map. The family of dilations $\left\{\delta_{\lambda}(x): \lambda>0\right\}$ on the Carnot group is defined as

$$
\delta_{\lambda} x=\delta_{\lambda}\left(x_{i j}\right)=\left(\lambda x_{1}, \lambda^{2} x_{2}, \ldots, \lambda^{m} x_{m}\right),
$$

where $x_{i}=\left(x_{i 1}, \ldots, x_{i n_{i}}\right)$. Moreover, $d\left(\delta_{\lambda} x\right)=\lambda^{Q} d x$ and the quantity $Q=$ $\sum_{i=1}^{m} i \operatorname{dim} V_{i}$ is called the homogeneous dimension of $\mathbb{G}$.

The Euclidean space $\mathbb{R}^{n}$ with the standard structure is an example of the Abelian Carnot group: the exponential map is the identity and the vector fields $\frac{\partial}{\partial x_{i}}, i=1, \ldots, n$ have only trivial commutators and form the basis of the corresponding Lie algebra.

The simplest example of a non-abelian Carnot group is the Heisenberg group $\mathbb{H}^{n}$. The non-commutative multiplication is defined as

$$
p q=(x, y, t)\left(x^{\prime}, y^{\prime}, t^{\prime}\right)=\left(x+x^{\prime}, y+y^{\prime}, t+t^{\prime}-2 x y^{\prime}+2 y x^{\prime}\right),
$$


where $x=\left(x_{1}, \ldots, x_{n}\right), y=\left(y_{1}, \ldots, y_{n}\right) \in \mathbb{R}^{n}, t \in \mathbb{R}$, and the left translation $L_{p}(q)=p q$ is defined. The left-invariant vector fields

$$
\begin{aligned}
X_{i} & =\frac{\partial}{\partial x_{i}}+2 y_{i} \frac{\partial}{\partial t}, \quad i=1, \ldots, n \\
Y_{i} & =\frac{\partial}{\partial y_{i}}-2 x_{i} \frac{\partial}{\partial t}, \quad i=1, \ldots, n \\
T & =\frac{\partial}{\partial t}
\end{aligned}
$$

form the basis of the Lie algebra of the Heisenberg group. All non-trivial relations are of the form $\left[X_{i}, Y_{i}\right]=-4 T, i=1, \ldots, n$, and all other commutators vanish. Thus, the Heisenberg algebra has the dimension $2 n+1$ and splits into the direct sum $\mathcal{G}=V_{1} \oplus V_{2}$. The vector space $V_{1}$ is generated by the vector fields $X_{i}, Y_{i}, i=1, \ldots, n$, and the space $V_{2}$ is the one-dimensional center which is spanned by the vector field $T$.

We use the Carnot-Carathéodory metric based on the length of horizontal curves. An absolutely continuous map $\gamma:[0, b] \rightarrow \mathbb{G}$ is called a curve. A curve $\gamma:[0, b] \rightarrow \mathbb{G}$ is said to be horizontal if its tangent vector $\dot{\gamma}(s)$ lies in the horizontal tangent space $H T_{\gamma(t)}$, i.e. there exist functions $a_{j}(s), s \in[0, b]$, such that $\sum_{j=1}^{n_{1}} a_{j}^{2} \leq 1$ and

$$
\dot{\gamma}(s)=\sum_{j=1}^{n_{1}} a_{j}(s) X_{1 j}(\gamma(s))
$$

The result of [5] implies that one can connect two arbitrary points $x, y \in \mathbb{G}$ by a horizontal curve. We fix on $H T_{x}$ a quadratic form $\langle\cdot, \cdot\rangle$, so that the vector fields $X_{11}(x), \ldots, X_{1 n_{1}}(x)$ are orthonormal with respect to this form at every $x \in \mathbb{G}$. Then the length of the horizontal curve $l(\gamma)$ is defined by the formula

$$
l(\gamma)=\int_{0}^{b}\langle\dot{\gamma}(s), \dot{\gamma}(s)\rangle^{1 / 2} d s=\int_{0}^{b}\left(\sum_{j=1}^{n_{1}}\left|a_{j}(s)\right|^{2}\right)^{1 / 2} d s .
$$

The Carnot-Carathéodory distance $d_{c}(x, y)$ is the infimum of the length over all horizontal curves connecting $x$ and $y \in \mathbb{G}$. Since the quadratic form is left-invariant, the Carnot-Carathéodory metric is also left-invariant. The group $\mathbb{G}$ is connected, therefore, the metric $d_{c}(x, y)$ is finite (see [19]). A homogeneous norm on $\mathbb{G}$ is, by definition, a continuous non-negative function $|\cdot|$ on $\mathbb{G}$, such that $|x|=\left|x^{-1}\right|,\left|\delta_{\lambda}(x)\right|=\lambda|x|$, and $|x|=0$, if and only if $x=0$. Since all homogeneous norms are equivalent, we choose one that 
satisfies the triangle inequality: $\left|x^{-1} y\right| \leq|x|+|y|$. By $B(x, r)$ we denote the open ball of radius $r>0$ centered at $x$ in the metric $d_{c}$. Note that $B(x, r)=\left\{y \in \mathbb{G}:\left|x^{-1} y\right|<r\right\}$ is the left translation of the ball $B(0, r)$ by $x$ which is the image of the "unit ball" $B(0,1)$ under $\delta_{r}$. The Hausdorff dimension of the metric space $\left(\mathbb{G}, d_{c}\right)$ coincides with its homogeneous dimension $Q$. By $|E|$ we denote the measure of the set $E$. Our normalizing condition is such that the balls of radius one have measure one:

$$
|B(0,1)|=\int_{B(0,1)} d x=1 .
$$

We have that $|B(0, r)|=r^{Q}$ because the Jacobian of the dilation $\delta_{r}$ is $r^{Q}$.

A curve $\gamma:[0, b] \rightarrow \mathbb{G}$ is called rectifiable if the supremum

$$
\sup \left\{\sum_{k=1}^{p-1} d_{c}\left(\gamma\left(t_{k}\right), \gamma\left(t_{k+1}\right)\right)\right\}
$$

is finite where the supremum ranges over all partitions $0=t_{1} \leq t_{2} \leq \ldots \leq$ $t_{p}=b$ of the segment $[0, b]$. We remark that the definition of a rectifiable curve is based on the Carnot-Carathéodory metric. That is why a curve is not rectifiable if it is not horizontal $[11,13]$. Thus, from now on, we work only with horizontal curves. A horizontal curve $\gamma$, which is rectifiable with respect to the Carnot-Carathéodory metric, is differentiable almost everywhere and the tangent vector $\dot{\gamma}$ belongs to $V_{1}$ (see [14]).

Let us define the $p$-module $M_{p}\left(\Gamma\left(K_{0}, K_{1} ; \Omega\right)\right)$ of the family of curves $\Gamma\left(K_{0}, K_{1} ; \Omega\right)$ and the $p$-capacity $\operatorname{cap}_{p}\left(K_{0}, K_{1} ; \Omega\right)$ on the Carnot group.

Our assumption is the following. Let $\langle a, b\rangle$ be an interval of one of the following types: $[a, b],[a, b),(a, b]$, or $(a, b)$. From now on, we suppose that a curve $\gamma:\langle a, b\rangle \rightarrow \mathbb{G}$ is parameterized by the length element. Let $\Omega$ be an open connected set (domain) on $\mathbb{G}, K_{0}$ and $K_{1}$ be closed nonempty disjoint sets in the closure $\bar{\Omega}$ of $\Omega$. We will call $\left(K_{0}, K_{1} ; \Omega\right)$ the condenser. We will use the symbol $\Gamma\left(K_{0}, K_{1} ; \Omega\right)$ to denote the family of curves $\gamma:\langle a, b\rangle \rightarrow \Omega \subset \mathbb{G}$ which connect the sets $K_{0}$ and $K_{1}$, namely, if $\gamma \in \Gamma\left(K_{0}, K_{1} ; \Omega\right)$, then $\overline{\gamma(\langle a, b\rangle)} \cap K_{i} \neq \emptyset, i=0,1$, and $\gamma(t) \in \Omega, t \in(a, b)$.

Let $\mathcal{F}\left(\Gamma\left(K_{0}, K_{1} ; \Omega\right)\right)$ denote the set of Borel functions $\rho: \Omega \rightarrow[0 ; \infty]$, such that for every locally rectifiable $\gamma \in \Gamma\left(K_{0}, K_{1} ; \Omega\right)$ we have

$$
\sup \int_{\gamma^{\prime}} \rho d s=\sup \int_{0}^{l\left(\gamma^{\prime}\right)} \rho\left(\gamma^{\prime}(t)\right) d t \geq 1 .
$$

The supremum is taken over all arcs $\gamma^{\prime}$, such that $\gamma^{\prime}=\left.\gamma\right|_{[\alpha, \beta]} \rightarrow \Omega,[\alpha, \beta] \subset$ $\langle a, b\rangle$ and $l\left(\gamma^{\prime}\right)$ is the length of $\gamma^{\prime}$. The quantity $\mathcal{F}\left(\Gamma\left(K_{0}, K_{1} ; \Omega\right)\right)$ is called the set of admissible densities for $\Gamma\left(K_{0}, K_{1} ; \Omega\right)$. 
Definition 1.1 Let $p \in(1, \infty)$. The quantity

$$
M_{p}\left(\Gamma\left(K_{0}, K_{1} ; \Omega\right)\right)=\inf \int_{\Omega} \rho^{p} d x
$$

is called the $p$-module of the family of curves $\Gamma\left(K_{0}, K_{1} ; \Omega\right)$. The infimum is taken over all functions $\rho \in \mathcal{F}\left(\Gamma\left(K_{0}, K_{1} ; \Omega\right)\right)$.

The Vitali-Carathéodory theorem [15] about approximation of a function from $L_{p}$ implies that the set of admissible densities can be reduced to the set of Borel lower semicontinuous functions. Hence, without loss of generality, we can assume that $\rho \in \mathcal{F}\left(\Gamma\left(K_{0}, K_{1} ; \Omega\right)\right)$ is semicontinuous in $\mathbb{G}$.

The properties of the module of a family of curves in the case of $\mathbb{G}=\mathbb{R}^{n}$ one can find, for instance, in [7].

If there exists a constant $L$ such that $|\varphi(x)-\varphi(y)| \leq L d_{c}(x, y)$ for all $x, y \in D, D \subset \subset \Omega$, then the function $\varphi: \Omega \rightarrow \mathbb{R}$ is called locally Lipschitz continuous in $\Omega \subset \mathbb{G}$. The Sobolev space $L_{p}^{1}(\Omega)$ over the domain $\Omega$ is defined as a completion of the class of locally Lipschitz continuous function with respect to the seminorm

$$
\left\|\varphi \mid L_{p}^{1}(\Omega)\right\|=\left(\int_{\Omega}\|X \varphi\|^{p} d x\right)^{1 / p}<\infty .
$$

Here $X \varphi=\left(X_{11} \varphi, \ldots, X_{1 n_{1}} \varphi\right)$ is called the horizontal gradient of $\varphi$ and $\|X \varphi\|=\left(\sum_{j=1}^{n_{1}}\left|X_{1 j} \varphi\right|^{2}\right)^{1 / 2}$. Thus, if $u$ is a smooth function, then $X u$ is a horizontal component of the usual Riemannian gradient of $u$.

Let $\mathcal{A}\left(K_{0}, K_{1} ; \Omega\right)$ denote the set of non-negative real valued, locally Lipschitz continuous functions $\varphi \in L_{p}^{1}(\Omega) \cap C(\Omega)$, such that $\varphi(x)=0(\varphi(x) \geq 1)$ in a neighborhood of $K_{0}\left(K_{1}\right)$.

Definition 1.2 For $p \in(1, \infty)$ we define the $p$-capacity of $\left(K_{0}, K_{1} ; \Omega\right)$ by

$$
\operatorname{cap}_{p}\left(K_{0}, K_{1} ; \Omega\right)=\inf \int_{\Omega}\|X \varphi\|^{p} d x
$$

where the infimum is taken over all $\varphi \in \mathcal{A}\left(K_{0}, K_{1} ; \Omega\right)$.

Our main result is the following theorem.

Theorem. Let $\Omega$ be a bounded domain in the Carnot group $\mathbb{G}$. Suppose that $K_{0}$ and $K_{1}$ are disjoint non-empty compact sets in the closure of $\Omega$. Then

$$
M_{p}\left(\Gamma\left(K_{0}, K_{1} ; \Omega\right)\right)=\operatorname{cap}_{p}\left(K_{0}, K_{1} ; \Omega\right) .
$$




\section{Preliminary results}

We define an absolutely continuous function on curves of the horizontal fibration. For this we consider a family of horizontal curves $\mathcal{Y}$ which form a smooth fibration of an open set $D \subset \mathbb{G}$. Usually, a curve $\gamma \in \mathcal{Y}$ is an orbit of a smooth horizontal vector field $Y \in V_{1}$. If we denote by $\varphi_{s}$ the flow associated with this vector field, then the fiber is of the form $\gamma(s)=\varphi_{s}(p)$. Here the point $p$ belongs to the surface $S$ which is transversal to the vector field $Y$. The parameter $s$ ranges over an open interval $J \in \mathbb{R}$. One can assume that there is a measure $d \gamma$ on the fibration $\mathcal{Y}$ of the set $D \subset \mathbb{G}$. The measure $d \gamma$ satisfies the inequalities

$$
k_{0}|B(x, R)|^{\frac{Q-1}{Q}} \leq \int_{\gamma \in \mathcal{Y}, \gamma \cap B(x, R) \neq \emptyset} d \gamma \leq k_{1}|B(x, R)|^{\frac{Q-1}{Q}}
$$

for a sufficiently small ball $B(x, R) \subset D$ with constants $k_{0}, k_{1}$ which do not depend on the ball $B(x, R)$ (see $[12,20,22])$.

Definition 2.1 A function $u: \Omega \rightarrow \mathbb{R}, \Omega \subset \mathbb{G}$, is said to be absolutely continuous on lines $(u \in A C L(\Omega))$ if for any domain $U, \bar{U} \subset \Omega$ and any fibration $\mathcal{X}$ defined by a left-invariant vector field $X_{1 j}, j=1, \ldots, n_{1}$, the function $u$ is absolutely continuous on $\gamma \cap U$ with respect to the $\mathcal{H}^{1}$-Hausdorff measure for $d \gamma$-almost all curves $\gamma \in \mathcal{X}$.

For such a function $u$ the derivatives $X_{1 j} u, j=1, \ldots, n_{1}$, exist almost everywhere in $\Omega$. If they belong to $L_{p}(\Omega)$ for all $X_{1 j} \in V_{1}$, then $u$ is said to be in $A C L^{p}(\Omega)$. If the function $f$ belongs to $L_{p}^{1}(\Omega)$, then there exists a function $u \in A C L^{p}(\Omega)$ such that $f=u$ almost everywhere.

The following lemma and theorem are reformulations of the well known result by Fuglede [7] (see also [21]) for the Carnot group.

Lemma 2.2 Suppose $E$ is a Borel set on the Carnot group $\mathbb{G}$ and $g_{k}: E \rightarrow \mathbb{R}$ is a sequence of Borel functions which converges to a Borel function $g: E \rightarrow \mathbb{R}$ in $L_{p}(E)$. There is a subsequence $\left\{g_{k_{j}}\right\}$, such that the equality

$$
\lim _{j \rightarrow \infty} \int_{\gamma}\left|g_{k_{j}}-g\right| d s=0
$$

holds for all rectifiable horizontal curves $\gamma \subset E$ except for some family whose p-module vanishes. 
We will prove the next theorem for completeness.

Theorem 2.1 Let $\Omega$ be an open subset of $\mathbb{G}$, and $u: \Omega \rightarrow \mathbb{R}$ be a function from $A C L^{p}(\Omega), p \in(1, \infty)$. The function $u$ is absolutely continuous on rectifiable closed parts of horizontal curves, except for a family of horizontal curves whose p-module vanishes.

Proof: Let $U_{l}$ be a sequence of open sets, such that $\bar{U}_{0} \subset \cdots \subset \bar{U}_{l} \subset \cdots \subset \Omega$, $\bigcup_{l=0}^{\infty} U_{l}=\Omega$. Denote by $\Gamma$ the family of locally rectifiable horizontal curves whose trace lies in $\Omega$, and such that the function $u$ is not absolutely continuous on each curve of $\Gamma$. By $\Gamma_{l}$ we denote the family of closed arcs of the curves $\gamma \in \Gamma$ which intersect $U_{l}$. By the property of monotonicity of the $p$-module we deduce that

$$
M_{p}(\Gamma) \leq \sum_{l=1}^{\infty} M_{p}\left(\Gamma_{l}\right)
$$

The proof will be complete if we establish that $M_{p}\left(\Gamma_{l}\right)=0$ for any arbitrary index $l$. For a function $u$ satisfying the assertion of Theorem 2.1 there exists a sequence of the $C^{\infty}$-functions $u^{(i)}, i \in \mathbb{N}$, which converges to $u$ uniformly in $\bar{U}_{l}$ [6]. Moreover, the sequence $X_{1 k} u^{(i)}$ converges to $X_{1 k} u$ in $L_{p}(\Omega), k=1, \ldots, n_{1}$. By Lemma 2.2 we choose a subsequence (which we denote by the same symbol) $u^{(i)}$, such that

$$
\int_{\gamma}\left\|X_{1 k} u^{(i)}-X_{1 k} u\right\| d s \rightarrow 0 \quad \forall \quad k=1, \ldots, n_{1}
$$

for all rectifiable horizontal curves $\gamma:[0, b] \rightarrow U_{l}$ except for a family $\widetilde{\Gamma}$ whose $p$-module $M_{p}(\widetilde{\Gamma})$ vanishes. We show that $\Gamma_{l} \subset \widetilde{\Gamma}$. Suppose that there exists a rectifiable horizontal curve $\gamma \in \Gamma_{l} \backslash \widetilde{\Gamma}$. It is assumed that this curve is parameterized by its length element. Since the functions $u^{i}(\gamma(s))$ are absolutely continuous, the sequence of functions

$$
u^{(i)}(\gamma(s))=u^{(i)}(\gamma(0))+\int_{0}^{s}\left(\sum_{k=1}^{n_{1}} a_{k}(t) X_{1 k} u^{(i)}(\gamma(t))\right) d t
$$

is defined for any $s \in[0, b]$.

The sequence $u^{(i)}(\gamma(s))$ converges uniformly to the function $u(\gamma(s))$ as $i \rightarrow \infty$. Moreover, from (2.1) we deduce that

$$
u(\gamma(s))=u(\gamma(0))+\int_{0}^{s}\left(\sum_{k=1}^{n_{1}} a_{k}(t) X_{1 k} u(\gamma(t))\right) d t .
$$

Hence, the function $u$ is absolutely continuous, and we derive that $u$ is absolutely continuous on $\gamma(s)$. This contradicts $\gamma \in \Gamma_{l} \backslash \widetilde{\Gamma}$. 
In our next step we establish an approximation property for functions $f \in L_{p}(D)$ defined on an open set $D \neq \mathbb{G}$.

Lemma 2.3 Suppose that $D$ is a bounded domain on the Carnot group $\mathbb{G}$. Let $f \in L_{p}(D)$ and $\varepsilon>0$. Then there exists a continuous function $\tilde{f}$ such that

$$
\left\|f-\tilde{f} \mid L_{p}(D)\right\|<\varepsilon .
$$

Proof: Making use of the Whitney lemma we can find points $x_{1}, x_{2}, \ldots$ in $D$ and positive numbers $r_{1}, r_{2}, \ldots$, such that

(i) $B\left(x_{i}, r_{i}\right) \subset D$,

(ii) $D \subset \bigcup_{i} B\left(x_{i}, r_{i}\right)$,

(iii) $B\left(x_{i}, 2 r_{i}\right) \subset D$,

(iv) $\sum_{i} \chi_{B\left(x_{i}, 2 r_{i}\right)} \leq M$, with some number $M$ independent of the choice of the set $D$ and of the point $x \in D$.

Also we can suppose, that the radii of the balls do not exceed $1 / 2$.

Let $\left\{h_{1}(x), h_{2}(x), \ldots\right\}$ be a partition of unity on $D$ subordinate to the cover $\left\{B\left(x_{1}, r_{1}\right), B\left(x_{2}, r_{2}\right), \ldots\right\}: h_{i}(x) \geq 0, \operatorname{supp}\left(h_{i}(x)\right) \subset B\left(x_{i}, r_{i}\right)$, and $\sum_{i=1}^{\infty} h_{i}(x)=1$ for $x \in D$. Set $f_{i}(x)=h_{i} f(x)$. Then $f_{i}, i=1,2, \ldots$, satisfy the following condition: $\operatorname{supp} f_{i} \subset B\left(x_{i}, r_{i}\right), f_{i} \in L_{p}(\mathbb{G})$ and $f(x)=$ $\sum_{i=1}^{\infty} f_{i}(x)$ for $x \in D$.

We write $\varphi^{i}$ for the continuous function supported in the ball $B\left(x_{i}, 2 r_{i}\right)$ such that $\int_{B\left(x_{i}, 2 r_{i}\right)} \varphi^{i}(x) d x=1$. Let us consider the convolution

$$
\tilde{f}_{i}(x)=f_{i} \star \varphi_{t}^{i}(x)=\int_{\mathbb{G}} f_{i}(y) \varphi_{t}^{i}\left(y^{-1} x\right) d y=\int_{\mathbb{G}} f_{i}\left(x y^{-1}\right) \varphi_{t}^{i}(y) d y,
$$

where $\varphi_{t}^{i}(x)=t^{-Q} \varphi^{i}\left(\delta_{1 / t} x\right)$. It is known [6] that in this case the inequality

$$
\left\|\tilde{f}_{i}-f_{i} \mid L_{p}(\mathbb{G})\right\|<2^{-i} \varepsilon
$$

holds as $t \rightarrow 0$ for arbitrary $\varepsilon>0$.

Let us define $\tilde{f}(x)=\sum_{i=1}^{\infty} \tilde{f}_{i}(x)$. The continuity of $\tilde{f}$ and the inequality

$$
\int_{D}|\tilde{f}-f|^{p} d x \leq \sum_{i=1}^{\infty}\left\{\int_{\mathbb{G}}\left|\tilde{f}_{i}-f_{i}\right|^{p} d x\right\}^{1 / p}<\varepsilon
$$

yield the required approximation. 
Using similar argumentation as in [10], we prove the next lemma.

Lemma 2.4 Let $\mathcal{B} \subset \mathcal{F}\left(\Gamma\left(K_{0}, K_{1} ; \Omega\right)\right)$ consist of continuous functions on $\Omega \backslash\left(K_{0} \cup K_{1}\right)$. Then,

$$
M=\inf _{\rho \in \mathcal{B}} \int_{\Omega} \rho^{p}(x) d x=M_{p}\left(\Gamma\left(K_{0}, K_{1} ; \Omega\right)\right) .
$$

Proof: Let $\left\{B\left(x_{i}, r_{i}\right)\right\}$ be a cover of the domain $D=\Omega \backslash\left(K_{0} \cup K_{1}\right)$ chosen as in the previous lemma. We also use the notation $\rho=\sum_{i=1}^{\infty} \rho_{i}=\sum_{i=1}^{\infty} h_{i} \rho$, where $\left\{h_{i}\right\}$ is a partition of unity subordinate to $\left\{B\left(x_{i}, r_{i}\right)\right\}$. Then by Lemma 2.3 for $\varepsilon>0$ and $\rho \in \mathcal{F}\left(\Gamma\left(K_{0}, K_{1} ; \Omega\right)\right)$ we find continuous function $\tilde{\rho}$, such that

$$
\int_{\Omega \backslash\left(K_{0} \cup K_{1}\right)} \tilde{\rho}^{p}(x) d x<\varepsilon+M_{p}\left(\Gamma\left(K_{0}, K_{1} ; \Omega\right)\right) .
$$

We claim that $(1+\varepsilon) \tilde{\rho}$ is an admissible density for $\Gamma\left(K_{0}, K_{1} ; \Omega\right)$. If $\gamma$ belongs to $\Gamma\left(K_{0}, K_{1} ; \Omega\right)$, then

$$
1 \leq \int_{\gamma} \rho d s=\int_{\gamma} \sum_{i=1}^{\infty} \rho_{i} d s \leq \sum_{i=1}^{\infty} \int_{\gamma \cap B\left(x_{i}, 2 r_{i}\right)} \rho_{i} d s .
$$

Making use of the construction of approximation from Lemma 2.3 with parameters $t_{i}<\varepsilon, i=1,2, \ldots$, we get

$$
\begin{aligned}
\int_{\gamma} \tilde{\rho} d s & =\int_{\gamma} \sum_{i=1}^{\infty} \tilde{\rho}_{i} d s=\int_{\gamma} \sum_{i=1}^{\infty} \int_{\mathbb{G}} \rho_{i}\left(x y^{-1}\right) \varphi_{t_{i}}^{i}(y) d y d s \\
& =\int_{\gamma} \sum_{i=1}^{\infty} \int_{\mathbb{G}} \rho\left(x\left(\delta_{t_{i}} z\right)^{-1}\right) \varphi^{i}(z) d z d s \\
& =\sum_{i=1}^{\infty} \int_{B\left(x_{i}, 2 r_{i}\right)} \varphi^{i}(z) d z \int_{\gamma \cap B\left(x_{i}, r_{i}\right)} \rho_{i}\left(x\left(\delta_{t_{i}} z\right)^{-1}\right) d s(x) .
\end{aligned}
$$

We note that $\int_{B\left(x_{i}, 2 r_{i}\right)} \varphi^{i}(z) d z=1$ by definition. Let us denote by $\tilde{\gamma}$ the image of the curve $\gamma$ under the map $\gamma \rightarrow \gamma \cdot\left(\delta_{t} z\right)^{-1}$. We can choose a sufficiently small $t_{i}$, such that the image $\gamma \cap B\left(x_{i}, r_{i}\right)$ is contained in the ball $B\left(x_{i}, 2 r_{i}\right)$. Moreover, $\left.|\dot{\tilde{\gamma}}|=\mid \dot{\gamma} \cdot\left(\delta_{t_{i}} z\right)^{-1}\right) \mid$. Changing variables in the last integral of (2.4), we obtain

$$
\begin{aligned}
\int_{\gamma} \tilde{\rho} d s & =\sum_{i=1}^{\infty} \int_{\tilde{\gamma} \cap B\left(x_{i}, 2 r_{i}\right)} \rho_{i}(y)|\dot{\tilde{\gamma}}|^{-1} d s(y) \\
& \geq \sum_{i=1}^{\infty} \int_{\tilde{\gamma} \cap B\left(x_{i}, 2 r_{i}\right)} \rho_{i}(y)\left(|\dot{\gamma}|+t_{i}|z|\right)^{-1} d s(y) \geq(1+\varepsilon)^{-1} .
\end{aligned}
$$


In the latter we used the inequalities

$$
|\dot{\tilde{\gamma}}| \leq|\dot{\gamma}|+t_{i}|z|, \quad|\dot{\gamma}| \leq 1, \quad t_{i}<\varepsilon, \quad|z|<2 r_{i}<1, \quad i=1,2, \ldots
$$

Since $\varepsilon$ and $\rho \in \Gamma\left(K_{0}, K_{1} ; \Omega\right)$ are arbitrary, we get from (2.3) that

$$
M=\inf _{\rho \in \mathcal{B}} \int_{\Omega} \rho^{p}(x) d x \leq M_{p}\left(\Gamma\left(K_{0}, K_{1} ; \Omega\right)\right) .
$$

The reverse inequality is obvious and we have (2.2) as desired.

\section{Proof of the main result}

In this section we will be working under the assumption that $K_{0}$ and $K_{1}$ are disjoint non-empty compacts in the closure $\bar{\Omega}$ of a bounded domain $\Omega \subset \mathbb{G}$. Moreover, let $K_{0}^{j}$ and $K_{1}^{j}$ be a sequence of closed sets, such that $K_{0}^{0} \cap K_{1}^{0}=\emptyset$, $K_{0}^{j} \subset \operatorname{int} K_{0}^{j-1}, K_{1}^{j} \subset \operatorname{int} K_{1}^{j-1}, K_{0}=\bigcap_{j=0}^{\infty} K_{0}^{j}$, and $K_{1}=\bigcap_{j=0}^{\infty} K_{1}^{j}$.

The next lemma in the particular case $\mathbb{G}=\mathbb{R}^{n}$ goes back to the work $[16]$ and, then is digested by Ohtsuka (see for instance [1]).

Lemma 3.1 Let $\rho \in L_{p}(\mathbb{G})$ be a positive function which is continuous in $\Omega \backslash\left(K_{0} \cup K_{1}\right)$. For each $\varepsilon>0$ we can construct a function $\rho^{\prime}$ on $\Omega, \rho^{\prime} \geq \rho$, with the following properties:

(i) $\int_{\Omega} \rho^{p} d x \leq \int_{\Omega} \rho^{p} d x+\varepsilon$.

(ii) Suppose that for each $j$ there is $\gamma_{j} \in \Gamma\left(K_{0}^{j}, K_{1}^{j} ; \Omega\right)$ such that $\int_{\gamma_{j}} \rho^{\prime} d s \leq \alpha$. Then there exists $\tilde{\gamma} \in \Gamma\left(K_{0}, K_{1} ; \Omega\right)$, such that $\int_{\tilde{\gamma}} \rho d s \leq \alpha+\varepsilon$.

Proof: The most difficult part of the lemma is the existence of $\tilde{\gamma}$ inside $\Omega$. It is rather easy to find a curve in $\bar{\Omega}$, but such a curve is not necessarily from $\Gamma\left(K_{0}, K_{1} ; \Omega\right)$.

For the beginning let us construct the function $\rho^{\prime}$. Let $K^{j}=K_{0}^{j} \cup K_{1}^{j}$, $W^{j}=K^{j-1} \backslash \operatorname{int} K^{j}$, and $d_{j}=\operatorname{dist}\left(\partial K^{j-1}, \partial K^{j}\right)>0$. Since the function $\rho$ is strictly positive, we can find a sequence $\varepsilon_{j} \rightarrow 0$, such that

$$
\begin{array}{r}
\sum_{j=1}^{\infty}\left(1+\varepsilon_{j}^{-1}\right)^{p} \varepsilon_{j}<\varepsilon, \\
\left(1+\varepsilon_{j}^{-1}\right) d_{j} \inf _{x \in W^{j} \cap \Omega} \rho(x)>\alpha .
\end{array}
$$

We can find a sequence of compact subsets $\Omega_{j} \subset \Omega$ increasing to $\Omega$, such that

$$
\int_{\Omega \backslash \Omega_{j}} \rho^{p} d x<\varepsilon_{j} .
$$


Let $V^{j}=\left(\Omega \backslash \Omega_{j}\right) \cap W^{j}$, and set

$$
\rho^{\prime}(x)=\left\{\begin{array}{cl}
\left(1+\varepsilon_{j}^{-1}\right) \rho(x) & \text { if } x \in V^{j} \\
\rho(x) & \text { if } x \in \Omega \backslash\left(\cup V^{j}\right) .
\end{array}\right.
$$

Now, applying (3.1), we obtain

$$
\begin{aligned}
\int_{\Omega} \rho^{\prime p} d x & =\sum_{j} \int_{V^{j}}\left(\left(1+\varepsilon_{j}^{-1}\right) \rho(x)\right)^{p} d x+\int_{\Omega \backslash\left(\cup V^{j}\right)} \rho^{p} d x \\
& \leq \sum_{j}\left(1+\varepsilon_{j}^{-1}\right)^{p} \int_{V^{j}} \rho^{p} d x+\int_{\Omega} \rho^{p} d x \\
& \leq \sum_{j}\left(1+\varepsilon_{j}^{-1}\right)^{p} \varepsilon_{j}+\int_{\Omega} \rho^{p} d x<\varepsilon+\int_{\Omega} \rho^{p} d x .
\end{aligned}
$$

We see that (i) holds. Now let us show (ii). Fix $j \geq 1$. The curve $\gamma_{k}$ is from $\Gamma\left(K_{0}^{j}, K_{1}^{j} ; \Omega\right)$ for the $k \geq j$ by definition. Hence, $\gamma_{k}$ contains two arcs: $\gamma_{k}^{\prime}$ such that $\gamma_{k}^{\prime}$ connects $\partial K_{0}^{j}$ and $\partial K_{0}^{j-1}$; and $\gamma_{k}^{\prime \prime}$ which connects $\partial K_{1}^{j}$ and $\partial K_{1}^{j-1}$. Let us show that $\gamma_{k}^{\prime}$ and $\gamma_{k}^{\prime \prime}$ are not included in $V^{j}$. On the contrary, let us suppose that $\gamma_{k}^{\prime} \subset V^{j}$. Then, using (3.2), we deduce the inequality

$\alpha \geq \int_{\gamma_{k}} \rho^{\prime} d s \geq \int_{\gamma_{k}^{\prime}} \rho^{\prime} d s \geq\left(1+\varepsilon_{j}^{-1}\right) \int_{\gamma_{k}^{\prime}} \rho d s \geq\left(1+\varepsilon_{j}^{-1}\right) \inf _{x \in W^{j} \cap \Omega} \rho(x) \int_{\gamma_{k}^{\prime}} d s>\alpha$,

which is false. In the same way $\gamma_{k}^{\prime \prime}$ is not included in $V^{j}$, therefore,

$$
\gamma_{k} \bigcap\left(\Omega_{j} \cap\left(K_{i}^{j-1} \backslash \operatorname{int} K_{i}^{j}\right)\right) \neq \emptyset \quad \text { for } \quad i=0,1 \quad \text { and } \quad k \geq j .
$$

Observe that the sets $\Omega_{j} \cap\left(K_{i}^{j-1} \backslash \operatorname{int} K_{i}^{j}\right), i=0,1$, are compacts. For a fixed $j$ let us consider a sequence $\left\{\gamma_{k}^{j}\right\}_{k=j}^{\infty}$. We can extract a subsequence (we use the same notation $\left\{\gamma_{k}^{j}\right\}_{k \rightarrow \infty}$ ) which converges to a curve $\gamma_{0}^{j}$, such that

$$
\gamma_{0}^{j} \bigcap\left(\Omega_{j} \cap\left(K_{0}^{j-1} \backslash \operatorname{int} K_{0}^{j}\right)\right) \neq \emptyset
$$

Further, we fix a point $x_{0}^{j} \in \Omega_{j} \cap\left(K_{0}^{j-1} \backslash \operatorname{int} K_{0}^{j}\right)$ on it. Since $\rho$ is continuous at $x_{0}^{j} \in \Omega_{j} \cap\left(K_{0}^{j-1} \backslash \operatorname{int} K_{0}^{j}\right)$, we can choose a ball $B\left(x_{0}^{j}, r\left(x_{0}^{j}\right)\right) \subset$ $\Omega$ so small that

$$
\int_{l} \rho d s \leq \varepsilon / 2^{j+3}
$$

for any shortest curve $l \subset B\left(x_{0}^{j}, r\left(x_{0}^{j}\right)\right)$ which connects the center $x_{0}^{j}$ with the boundary of $B\left(x_{0}^{j}, r\left(x_{0}^{j}\right)\right)$. Renumbering the subsequence, we may assume that each member of the subsequence $\left\{\gamma_{k}^{j}\right\}$ intersects $B\left(x_{0}^{j}, r\left(x_{0}^{j}\right)\right)$. 
In the same way we can find a small closed ball $B\left(x_{1}^{j}, r\left(x_{1}^{j}\right)\right) \subset \Omega, x_{1}^{j} \in$ $\Omega_{j} \cap\left(K_{1}^{j-1} \backslash \operatorname{int} K_{1}^{j}\right)$, so that $\left\{\gamma_{k}^{j}\right\}$ intersects $B\left(x_{1}^{j}, r\left(x_{1}^{j}\right)\right)$ and an analogue of (3.3) holds. We start this process from $j=1$ and extract a subsequence $\left\{\gamma_{k}^{j}\right\}$ from the sequence constructed in the previous step, such that the new subsequence $\left\{\gamma_{k}^{j}\right\}$ intersects $B\left(x_{0}^{j}, r\left(x_{0}^{j}\right)\right)$ and $B\left(x_{1}^{j}, r\left(x_{1}^{j}\right)\right)$.

Now let us consider the diagonal $\left\{\gamma_{k}^{k}\right\}$. Then $\left\{\gamma_{k}^{k}\right\}$ intersects $B\left(x_{0}^{j}, r\left(x_{0}^{j}\right)\right)$ and $B\left(x_{1}^{j}, r\left(x_{1}^{j}\right)\right)$ for $1 \leq j \leq k$. In each ball $B\left(x_{i}^{j}, r\left(x_{i}^{j}\right)\right), i=0,1$, we add two shortest curves to $\left\{\gamma_{k}^{k}\right\}$ connecting $x_{i}^{j}$ with the points of intersections of $\left\{\gamma_{k}^{k}\right\}$ with $\partial B\left(x_{i}^{j}, r\left(x_{i}^{j}\right)\right), i=0,1,1 \leq j \leq k$. Thus, we have a connected horizontal curve $\tilde{\gamma}_{k} \in \Gamma\left(K_{0}^{k}, K_{1}^{k} ; \Omega\right)$ passing through all pairs $\left\{x_{0}^{j}, x_{1}^{j}\right\}_{j=1}^{k}$. We have by (3.3) that

$$
\int_{\tilde{\gamma}_{k}} \rho d s \leq \int_{\gamma_{k}^{k}} \rho d s+2 \sum_{j=1}^{k} \frac{\varepsilon}{2^{j+3}} \leq \alpha+\frac{\varepsilon}{4} .
$$

Let $\Gamma_{0}$ be the union of all horizontal curves in $\Omega \backslash\left(K_{0} \cup K_{1}\right)$ connecting $x_{0}^{1}$ and $x_{1}^{1}$. For $i=0,1$, let $\Gamma_{i}^{j}$ be the collection of all horizontal curves in $\Omega \backslash\left(K_{0} \cup K_{1}\right)$ connecting $x_{i}^{j}$ and $x_{i}^{j+1}$. Then,

$$
\inf _{\gamma \in \Gamma_{0}} \int_{\gamma} \rho d s+\sum_{j=1}^{k} \inf _{\gamma \in \Gamma_{0}^{j}} \int_{\gamma} \rho d s+\sum_{j=1}^{k} \inf _{\gamma \in \Gamma_{1}^{j}} \int_{\gamma} \rho d s \leq \int_{\tilde{\gamma}_{k}} \rho d s \leq \alpha+\frac{\varepsilon}{4} .
$$

Therefore, we can choose $C_{0} \in \Gamma_{0}$ and $C_{i}^{j} \in \Gamma_{i}^{j}$, such that

$$
\begin{aligned}
& \int_{C_{0}} \rho d s<\inf _{\gamma \in \Gamma_{0}} \int_{\gamma} \rho d s+\frac{\varepsilon}{2}, \\
& \int_{C_{i}^{j}} \rho d s<\inf _{\gamma \in \Gamma_{i}^{j}} \int_{\gamma} \rho d s+\frac{\varepsilon}{2^{j+3}} .
\end{aligned}
$$

Let

$$
\tilde{\gamma}=\ldots+C_{0}^{1}+C_{0}+C_{1}^{1}+\ldots
$$

Then, $\tilde{\gamma} \in \Gamma\left(K_{0}, K_{1} ; \Omega\right)$ and

$$
\int_{\tilde{\gamma}} \rho d s \leq \alpha+\frac{\varepsilon}{4}+\frac{\varepsilon}{2}+2 \sum_{j=1}^{\infty} \frac{\varepsilon}{2^{j+3}}=\alpha+\varepsilon .
$$

The lemma is proved. 
Theorem 3.1 Let $\Omega$ be a bounded domain in the Carnot group $\mathbb{G}$. Suppose $K_{0}$ and $K_{1}$ to be disjoint non-empty compacts in the closure of $\Omega$. Then,

$$
M_{p}\left(\Gamma\left(K_{0}, K_{1} ; \Omega\right)\right)=\operatorname{cap}_{p}\left(K_{0}, K_{1} ; \Omega\right) .
$$

Proof: Our proof falls into three steps.

Step 1. We start proving the inequality

$$
M_{p}\left(\Gamma\left(K_{0}, K_{1} ; \Omega\right)\right) \leq \operatorname{cap}_{p}\left(K_{0}, K_{1} ; \Omega\right)
$$

Let $u \in \mathcal{A}\left(K_{0}, K_{1} ; \Omega\right)$. Let $\Gamma_{0}$ be the locally rectifiable horizontal curves $\gamma \in \Gamma\left(K_{0}, K_{1} ; \Omega\right)$, such that $u$ is absolutely continuous on every rectifiable closed part of $\gamma$. Define $\rho: \Omega \rightarrow[0, \infty]$ by

$$
\rho(x)=\left\{\begin{array}{cl}
\|X u\| & \text { if } x \in \Omega, \\
0 & \text { if } x \notin \Omega .
\end{array}\right.
$$

Suppose that $\gamma \in \Gamma_{0}$ and $\gamma:(a, b) \rightarrow \Omega$ is parameterized by the length element. If $a<t_{1}<t_{2}<b$, then making use of the inequality $|\dot{\gamma}(t)| \leq 1$, we get

$$
\begin{aligned}
\int_{\gamma} \rho d s & =\int_{a}^{b} \rho(\gamma(t)) d t \geq \int_{t_{1}}^{t_{2}}\|X u(\gamma(t))\| d t \\
& \geq\left|\int_{t_{1}}^{t_{2}}\langle X u(\gamma(t)) \cdot \dot{\gamma}(t)\rangle d t\right|=\left|u\left(\gamma\left(t_{2}\right)\right)-u\left(\gamma\left(t_{1}\right)\right)\right| .
\end{aligned}
$$

Since $t_{1}$ and $t_{2}$ are arbitrary, (3.5) implies the inequality $\int_{\gamma} \rho d s \geq 1$. Hence, $\rho$ is admissible for the family of curves $\Gamma\left(K_{0}, K_{1} ; \Omega\right)$. Therefore,

$$
M_{p}\left(\Gamma_{0}\right) \leq \int_{\Omega} \rho^{p}(x) d x=\int_{\Omega}\|X u(x)\|^{p} d x .
$$

Taking infimum over all $u \in \mathcal{A}\left(K_{0}, K_{1} ; \Omega\right)$ we get $M_{p}\left(\Gamma_{0}\right) \leq \operatorname{cap}_{p}\left(K_{0}, K_{1} ; \Omega\right)$. Theorem 2.1 implies $M_{p}\left(\Gamma_{0}\right)=M_{p}\left(\Gamma\left(K_{0}, K_{1} ; \Omega\right)\right)$, and (3.4) follows from the above.

Step 2. Now we prove the reverse inequality

$$
M_{p}\left(\Gamma\left(K_{0}, K_{1} ; \Omega\right)\right) \geq \operatorname{cap}_{p}\left(K_{0}, K_{1} ; \Omega\right)
$$

for the case $\left(K_{0} \cup K_{1}\right) \cap \partial \Omega=\emptyset$. Lemma 2.4 allows us to assume that $\rho \in$ $\mathcal{F}\left(\Gamma\left(K_{0}, K_{1} ; \Omega\right)\right)$ is continuous in $\Omega \backslash\left(K_{0} \cup K_{1}\right)$. Let us define $u: \Omega \rightarrow[0, \infty]$ by $u(x)=\min \left(1, \inf \int_{\beta_{x}} \rho d s\right)$ where the infimum is taken over all locally rectifiable horizontal curves $\beta_{x} \in \Omega$ connecting $K_{0}$ and $x$. We claim that $u \in \mathcal{A}\left(K_{0}, K_{1} ; \Omega\right)$ and $\|X u\| \leq \rho$ almost everywhere in $\Omega$. If $u \equiv 1$, then there is nothing to prove. 
Let $u \not \equiv 1$, and let $\alpha_{x_{1}, x_{2}}$ be a shortest curve which connect $x_{1}$ and $x_{2}$, and $\beta_{x_{1}}$ be a rectifiable curve connecting $K_{0}$ and $x_{1}$. Then,

$$
u\left(x_{2}\right) \leq \int_{\beta_{x_{1}}} \rho d s+\int_{\alpha_{x_{1}, x_{2}}} \rho d s \leq \int_{\beta_{x_{1}}} \rho d s+\max _{x \in \alpha_{x_{1}, x_{2}}} \rho(x) d_{c}\left(x_{1}, x_{2}\right) .
$$

Since $\beta_{x_{1}}$ is arbitrary, we obtain

$$
u\left(x_{2}\right) \leq u\left(x_{1}\right)+\max _{x \in \alpha_{x_{1}, x_{2}}} \rho(x) d_{c}\left(x_{1}, x_{2}\right) .
$$

Similarly, we have

$$
u\left(x_{1}\right) \leq u\left(x_{2}\right)+\max _{x \in \alpha_{x_{1}, x_{2}}} \rho(x) d_{c}\left(x_{1}, x_{2}\right) .
$$

These two inequalities prove that

$$
\left|u\left(x_{1}\right)-u\left(x_{2}\right)\right| \leq \max _{x \in \alpha_{x_{1}, x_{2}}} \rho(x) d_{c}\left(x_{1}, x_{2}\right) .
$$

If $u$ satisfies (3.7), then $u$ is locally Lipschitz continuous in $\Omega$. Therefore, $u$ has the derivative $X_{1 j} u, j=1, \ldots, n_{1}$, almost everywhere in $\Omega$ by [14]. Suppose now that $x_{0} \in \Omega$ is a point where the derivatives $X_{1 j} u, j=1, \ldots, n_{1}$ exist, then we get

$$
\left|u\left(x_{0} h\right)-u\left(x_{0}\right)\right|=|h||| X u\left(x_{0}\right)||+o(|h|) \leq \max _{x \in \alpha_{x_{0}, x_{0} h}} \rho(x)|h| .
$$

Letting $|h| \rightarrow 0$, we obtain $\left\|X u\left(x_{0}\right)\right\| \leq \rho\left(x_{0}\right)$. Therefore,

$$
\operatorname{cap}_{p}\left(K_{0}, K_{1} ; \Omega\right) \leq \int_{\Omega}\|X u\|^{p} d x \leq \int_{\Omega} \rho^{p} d x
$$

and (3.6) holds.

By (3.4) and (3.6) we conclude that, if $\left(K_{0} \cup K_{1}\right) \cap \partial \Omega=\emptyset$, then

$$
\operatorname{cap}_{p}\left(K_{0}, K_{1} ; \Omega\right)=M_{p}\left(\Gamma\left(K_{0}, K_{1} ; \Omega\right)\right) .
$$

Step 3. Fix $\varepsilon \in(0,1 / 2)$ and let $\left(K_{0} \cup K_{1}\right) \cap \partial \Omega \neq \emptyset$. Let $\rho \in$ $\mathcal{F}\left(\Gamma\left(K_{0}, K_{1} ; \Omega\right)\right)$ be a continuous function in $\Omega \backslash\left(K_{0} \cup K_{1}\right)$, such that

$$
\int_{\Omega \backslash\left(K_{0} \cup K_{1}\right)} \rho^{p} d x<\varepsilon+M_{p}\left(\Gamma\left(K_{0}, K_{1} ; \Omega\right)\right) .
$$

We may assume that $\rho$ is strictly positive on $\Omega \backslash\left(K_{0} \cup K_{1}\right)$. If this were not so, we could consider the cut-of-function $\max (\rho, 1 / m)$ instead of $\rho$ and suppose that this function satisfies the inequality

$$
\int_{\Omega \backslash\left(K_{0} \cup K_{1}\right)}(\max (\rho, 1 / m))^{p} d x<\varepsilon+M_{p}\left(\Gamma\left(K_{0}, K_{1} ; \Omega\right)\right)
$$

for a sufficiently big $m \in \mathbb{N}$. 
Let $\rho^{\prime},\left\{K_{0}^{j}\right\},\left\{K_{1}^{j}\right\}$, be as in Lemma 3.1. We show that

$$
\int_{\gamma} \rho^{\prime} d s>1-2 \varepsilon \quad \text { for all } \quad \gamma \in \Gamma\left(K_{0}^{j}, K_{1}^{j} ; \Omega\right)
$$

for a sufficiently big $j \in \mathbb{N}$. In fact, if we supposed the contrary, there would be a sequence $\left\{j_{k}\right\}$ and curves $\gamma_{k} \in \Gamma\left(K_{0}^{j_{k}}, K_{1}^{j_{k}} ; \Omega\right)$, such that

$$
\int_{\gamma_{k}} \rho^{\prime} d s \leq 1-2 \varepsilon .
$$

By Lemma 3.1 we would find $\tilde{\gamma} \in \Gamma\left(K_{0}, K_{1} ; \Omega\right)$, such that

$$
\int_{\tilde{\gamma}} \rho d s \leq 1-2 \varepsilon+\varepsilon=1-\varepsilon,
$$

which contradicts $\rho \in \mathcal{F}\left(\Gamma\left(K_{0}, K_{1} ; \Omega\right)\right)$.

Next we define the function

$$
\tilde{\rho}(x)=\left\{\begin{array}{cl}
\frac{\rho^{\prime}}{1-2 \varepsilon} & \text { if } x \in \Omega \backslash\left(K_{0}^{j} \cup K_{1}^{j}\right), \\
0 & \text { if } x \notin \Omega \backslash\left(K_{0}^{j} \cup K_{1}^{j}\right) .
\end{array}\right.
$$

It belongs to $\mathcal{F}\left(\Gamma\left(K_{0}, K_{1} ; \Omega \cup K_{0}^{j} \cup K_{1}^{j}\right)\right)$. This fact and the equality (3.8) for $\left(K_{0}, K_{1} ; \Omega \cup K_{0}^{j} \cup K_{1}^{j}\right)$ imply

$$
\begin{aligned}
& \left(M_{p}\left(\Gamma\left(K_{0}, K_{1} ; \Omega\right)\right)+2 \varepsilon\right)(1-2 \varepsilon)^{1-p} \geq \int_{\Omega} \tilde{\rho}^{p} d x \geq M_{p}\left(\Gamma\left(K_{0}, K_{1} ; \Omega \cup K_{0}^{j} \cup K_{1}^{j}\right)\right) \\
& =\operatorname{cap}_{p}\left(K_{0}, K_{1} ; \Omega \cup K_{0}^{j} \cup K_{1}^{j}\right) \geq \operatorname{cap}_{p}\left(K_{0}, K_{1} ; \Omega\right) .
\end{aligned}
$$

Hence, letting $j \rightarrow \infty$, and then $\varepsilon \rightarrow 0$, we obtain

$$
M_{p} \Gamma\left(\left(K_{0}, K_{1} ; \Omega\right)\right) \geq \operatorname{cap}_{p}\left(K_{0}, K_{1} ; \Omega\right)
$$

and the theorem is proved.

Theorem 3.2 Let $K_{0}$ and $K_{1}$ be disjoint non-empty closed sets in the closure $\bar{\Omega}$ of a bounded domain $\Omega \subset \mathbb{G}$. Let $K_{0}^{j}$ and $K_{1}^{j}$ be sequences of compact sets, such that $K_{0}^{0} \cap K_{1}^{0}=\emptyset, K_{0}^{j} \subset \operatorname{int} K_{0}^{j-1}, K_{1}^{j} \subset \operatorname{int} K_{1}^{j-1}, K_{0}=\bigcap_{j=0}^{\infty} K_{0}^{j}$, and $K_{1}=\bigcap_{j=0}^{\infty} K_{1}^{j}$. Then,

$$
M_{p}\left(\Gamma\left(K_{0}, K_{1} ; \Omega\right)\right)=\lim _{j \rightarrow \infty} M_{p}\left(\Gamma\left(K_{0}^{j}, K_{1}^{j} ; \Omega\right)\right) .
$$


Proof: Let $\rho \in \mathcal{F}\left(\Gamma\left(K_{0}, K_{1} ; \Omega\right)\right)$. Lemma 2.4 allows us to assume that $\rho$ is continuous in $\Omega \backslash\left(K_{0} \cup K_{1}\right)$. We fix $\varepsilon \in(0,1)$ and choose $\rho$, such that

$$
\int_{\Omega} \rho^{p} d x \leq M_{p}\left(\Gamma\left(K_{0}, K_{1} ; \Omega\right)\right)+\varepsilon
$$

For a function $\rho$ we can construct $\rho^{\prime}$ as in Lemma 3.1. Moreover, $(1-$ $2 \varepsilon)^{-1} \rho^{\prime} \in \mathcal{F}\left(\Gamma_{j}\left(K_{0}^{j}, K_{1}^{j} ; \Omega\right)\right)$ as it was shown in the proof of the step 3 of Theorem 3.1. From all these facts we deduce

$M_{p}\left(\Gamma\left(K_{0}^{j}, K_{1}^{j} ; \Omega\right)\right) \leq \int_{\Omega}\left((1-2 \varepsilon)^{-1} \rho^{\prime}\right)^{p} d x \leq(1-2 \varepsilon)^{-p}\left(M_{p}\left(\Gamma\left(K_{0}, K_{1} ; \Omega\right)\right)+\varepsilon\right)$.

Hence, letting $j \rightarrow \infty$, and then $\varepsilon \rightarrow 0$, we obtain the desired result.

\section{References}

[1] Aikawa, H. And Ohtsuka, M.: Extremal length of vector measures. Ann. Acad. Scien. Fennicæ 24 (1999), 61-88.

[2] Caraman, P.: New cases of equality between $p$-module and $p$-capacity. Proceedings of the Tenth Conf. on analytic function, Szczyrk, 1990. Ann. Polon. Math. 55 (1991), 37-56.

[3] Caraman, P.: Relations between $p$-capacity and p-module. I. II. Rev. Roumaine Math. Pures Appl. 39 (1994), no. 6, 509-553, 555-577.

[4] Caraman, P.: The problem of equality between the $p$-capacity and p-module. Bull. Soc. Sci. Lett. Eódź Sér. Rech. Déform. 20 (1995), 79-89.

[5] Chow, W. L.: Systeme von linearen partiellen differential gleichungen erster ordnug. Math. Ann. 117 (1939), 98-105.

[6] Foland, G. B. And Stein, E. M.: Hardy spaces on homogeneous groups. Math. Notes 28 (1982), Princeton University Press, Princeton, New Jersey.

[7] Fuglede, B.: Extremal length and functional completion. Acta Math. 98 (1957), 171-219.

[8] Heinonen, J. and Koskela, P.: Quasikonformal maps in metric spaces with controlled geometry. Acta Math. 181 (1998), 1-61.

[9] HajŁasz, P. and Koskela, P.: Sobolev met Poincarè. Mem. Amer. Math. Soc. 145 (2000), no. 688, 101 pp.

[10] Hesse, J.: A p-extremal length and p-capacity equality. Ark. Mat. 13 (1975), no. 1, 131-144.

[11] KorÁnyi, A.: Geometric aspects of analysis on the Heisenberg group. Topic in Modern Harmonic Analysis. Instituto nazionale di Alta matematica, Roma, 1983.

[12] Korányi, A. And Reimann, H. M.: Foundation for the theory of quasiconformal mapping on the Heisenberg group. Adv. Math. 111 (1995), 1-87. 
[13] Mitchell, J.: On Carnot-Carathéodory metrics. J. Diff. Geom. 21 (1985), $35-45$.

[14] Pansu, P.: Métriques de Carnot-Carathéodory et quasiisométries des espacies symétriques de rang un. Ann. of Math. 129 (1989), 1-60.

[15] Rudin, W.: Real and complex analysis. McGraw-Hill Book Company, New York, 1966.

[16] Shlyk, V. A.: On the equality between $p$-capacity and $p$-modulus. Siberian Math. J. 34 (1993), no. 6, 1196-1200.

[17] Stein, E. M.: Some problems in harmonic analysis suggested by symmetric spaces and semisimple groups. Proc. Int. Congr. Math., Nice I, (1970), Gauthier-Villars, Paris, 1971, 173-179.

[18] Stein, E. M.: Harmonic analysis: real variable, methods, orthogonality and oscillatory integrals. Princeton Univ. Press, 1993.

[19] Stricharts, R. S.: Sub-Riemannian Geometry. J. Diff. Geom. 24 (1986), 221-263.

[20] Ukhlov, A. D. And Vodop'yanov, S. K.: Sobolev spaces and $P, Q-$ quasiconformal mappings of the Carnot groups. Siberian Math. J. 39 (1998), no. 4, 665-682.

[21] VÄIsÄLÄ, J.: Lectures on n-dimensional quasiconformal mapping. Lecture Notes in Math. 229. Springer-Verlag, Berlin, 1971.

[22] Vodop'yanov, S. K.: P-Differentiability on Carnot groups in different topologies and related topics. Proc. on Anal. and Geom. Novosibirsk: Sobolev Institute Press (2000), 603-670.

[23] Ziemer, W. P.: Extremal length and p-capacity. Michigan Math. J. 16 (1963), 43-51.

Recibido: 30 de julio de 2001

Revisado: 5 de diciembre de 2001

Irina Markina

Departamento de Matemática

Universidad Técnica Federico Santa María

Valparaíso, Chile

irina.markina@mat.utfsm.cl

This work was supported by Project Fondecyt (Chile) \# 1020067. 